\title{
Wave equation for sound in fluids with vorticity
}

\author{
Santiago Esteban Perez Bergliaffa ${ }^{\text {a }}$, Katrina Hibberd ${ }^{\text {, }}$, \\ Michael Stone $^{\mathrm{c}}$, Matt Visser ${ }^{\mathrm{d}, *}$ \\ ${ }^{a}$ Centro Brasileiro de Pesquisas Físicas, Rua Dr. Xavier Sigaud 150, 22290-180 Urca, Rio de Janeiro, RJ, Brazil \\ ${ }^{\mathrm{b}}$ GCEP, Center of Physics, Universidade do Minho, 4710-057 Braga, Portugal \\ ${ }^{\mathrm{c}}$ Physics Department, University of Illinois, 1110 W. Green Street, Urbana, IL 61801, USA \\ ${ }^{\mathrm{d}}$ School of Mathematical and Computing Sciences, Victoria University of Wellington, PO Box 600, Wellington, New Zealand
}

Received 28 May 2002; received in revised form 27 October 2003; accepted 7 November 2003

Communicated by C.K.R.T. Jones

\begin{abstract}
We use Clebsch potentials and an action principle to derive a complete closed system of gauge-invariant equations for sound superposed on a general background flow. Our system reduces to the Unruh [Phys. Rev. Lett. 46 (1981) 1351] and Pierce [J. Acoust. Soc. Am. 87 (1990) 2292] wave equations when the flow is irrotational, or slowly varying. We illustrate our formalism by applying it to waves propagating in a uniformly rotating fluid where the sound modes hybridize with inertial waves. (C) 2003 Elsevier B.V. All rights reserved.
\end{abstract}

PACS: 43.20.Bi; 43.28.Py

Keywords: General linear acoustics; Sound in flows

\section{Introduction}

Studies of sound in a stationary fluid usually start from the wave equation whose derivation appears in all elementary texts, for example see [1]. When the fluid is moving, however, finding an appropriate generalization of the wave equation is not always possible. An exception is the special circumstance that the equation of state is barotropic and the background flow irrotational, but not necessarily steady. In this case a particularly attractive equation was derived by Unruh [2,3]. Unruh's equation was later rediscovered and further popularized by Visser [4,5]. It coincides with the equation obeyed by a relativistic scalar field propagating in curved space-time. The space-time geometry is governed by the acoustic metric which depends on the background flow velocity and on the local fluid density and speed of sound. The curved space-time interpretation of the wave equation is rather more than a mathematical curiosity. As well providing an attractive analogy with some aspects of general relativity [6], one can use the geometric formalism for ray tracing, and to produce a straightforward and systematic derivation of various conservation laws associated with acoustic energy and momentum $[7,8]$.

\footnotetext{
* Corresponding author. Fax: +64-4-463-5045.

E-mail addresses: sepb@cbpf.br (S.E. Perez Bergliaffa), keh@ fisica.uninho.pt (K. Hibberd), m-stone5@uiuc.edu (M. Stone), matt.visser@mcs.vuw.ac.nz (M. Visser).
} 
Unfortunately most fluid motions occurring in nature are not irrotational. It is therefore desirable to explore the possibility of extending the acoustic metric equation to a wider class of flows. A valuable step in this direction was taken by Pierce [9] who, without assuming that the background flow was irrotational, derived an equation which is in appearance equivalent to the acoustic metric equation. He did, however, assume that the background flow varied slowly over the length and time scale of the sound wave. As a result of his approximations, his dependent variable is not quite the velocity potential appearing in the acoustic metric equation. It gives the fluctuating velocity only up to a correction whose magnitude depends on the space and time inhomogeneities of the background flow. Pierce did not attempt to characterize the correction beyond estimating its size and showing that it was small in most regimes of interest in acoustics. He did show, however, that in special cases his equation reduces to known exact wave equations. In particular, for steady irrotational flow it reduces to Blokhintsev's equation [10,11], which is a special case of Unruh's. This and other features led Pierce to conjecture that his equation is of wider applicability than his derivation suggests. The purpose of the present paper is to show that this conjecture is correct.

We use Clebsch potentials and an action principle to derive the equations of motion for small perturbations about a general barotropic flow. Our principal result is an exact and concise expression for the small correction to potential flow, and a simple equation of motion obeyed by it. This, coupled with the Pierce equation, provides a closed system for wave propagation in a general inhomogeneous and unsteady background flow. The condition that the correction to potential flow be ignorable, and thus the acoustic metric equation accurate, is that the frequency of the sound be appreciably higher than the local vorticity. There is no requirement that the spatial inhomogeneity be small. Even if the frequency condition is violated, we can still study the wave motion, but with a more complicated system of partial differential equations.

The paper is organized as follows: in Section 2 we provide a very brief four-equation outline of key results. Section 3 reviews the action principle for the Clebsch formulation of barotropic fluid mechanics. (The straightforward but messy derivation of the Euler equations is presented in Appendix A.) In Section 4 we consider first-order perturbations to a background flow, and identify two "gauge-invariant" combinations of the potentials which have physical significance. (Technical discussion of the infinite family of conserved quantities that generate global gauge transformations on the Clebsch potentials is deferred until Appendix B.) In Section 5 we derive a closed system of equations for these combinations, and in Section 6 present a physical interpretation for one of the perturbations in terms of the displacement field. Finally Section 7 illustrates our formalism by applying it to a well-understood situation: waves in a uniformly rotating fluid.

\section{Outline}

In brief: any vector field in three dimensions can be represented in Clebsch form:

$$
\mathbf{v}_{0}=\nabla \phi_{0}+\beta_{0} \nabla \gamma_{0} .
$$

Once this is done, fluctuations around this background vector field can be represented as

$$
\mathbf{v}_{1}=\nabla \phi_{1}+\beta_{0} \nabla \gamma_{1}+\beta_{1} \nabla \gamma_{0}=\nabla\left(\phi_{1}+\beta_{0} \gamma_{1}\right)-\gamma_{1} \nabla \beta_{0}+\beta_{1} \nabla \gamma_{0} \equiv \nabla \psi_{1}+\xi_{1} .
$$

A nice feature of this representation is that $\xi_{1} \cdot\left(\nabla \times \mathbf{v}_{0}\right)=0$. Now interpret $\mathbf{v}$ as the fluid velocity, in the body of the paper we will derive an exact closed system of coupled differential equations for the perturbation:

$$
\begin{aligned}
& \frac{\mathrm{d}}{\mathrm{d} t}\left(\frac{1}{c^{2}} \frac{\mathrm{d}}{\mathrm{d} t} \psi_{1}\right)=\frac{1}{\rho_{0}} \nabla\left(\rho_{0}\left(\nabla \psi_{1}+\xi_{1}\right)\right), \\
& \frac{\mathrm{d} \xi_{1}}{\mathrm{~d} t}=\nabla \psi_{1} \times \omega_{0}-\left(\xi_{1} \cdot \nabla\right) \mathbf{v}_{0} .
\end{aligned}
$$

Deriving, interpreting, and analyzing these coupled wave equations is the central theme of this article. 


\section{Clebsch representation}

In this section, we will review the Clebsch potential approach to fluid dynamics. The Clebsch formalism has the advantage that the equations of motion may be derived from an action principle [12], and with an action principle conservation laws are related to symmetries by Noether's theorem.

We begin with

$$
S=\int \mathrm{d} t \mathrm{~d}^{3} x\left\{-\frac{1}{2} \rho \mathbf{v}^{2}-\phi(\dot{\rho}+\nabla \cdot(\rho \mathbf{v}))+\rho \beta(\dot{\gamma}+(\mathbf{v} \cdot \nabla) \gamma)+u(\rho)\right\} .
$$

Here $\rho$ is the fluid mass-density, $\mathbf{v}$ the velocity, and $u(\rho)$ the internal energy density. This is the customary expression giving rise to irrotational fluid dynamics (see, for example [7]) — but with an additional term containing new fields: $\beta$ and $\gamma$. The variable $\beta$ may be thought of as a Lagrange multiplier enforcing the Lin constraint [13] that there be a label $(\gamma)$ painted on the particles permitting us to distinguish one from another. (Lin originally employed three Lagrange multipliers $\beta_{1,2,3}$ leading to the conservation of three Lagrange co-ordinates, $\gamma_{1,2,3}$, which served to label the material particles uniquely. As shown by Seligar and Whitham [14], only one of these Lagrange multipliers is really necessary.)

Requiring that $S$ be stationary when we vary $\mathbf{v}$ gives

$$
-\rho \mathbf{v}+\rho \nabla \phi+\rho \beta \nabla \gamma=0,
$$

or

$$
\mathbf{v}=\nabla \phi+\beta \nabla \gamma
$$

This is the Clebsch representation $[15,16]$ of the velocity field. It allows for flows with non-zero vorticity:

$$
\omega=\nabla \times \mathbf{v}=\nabla \beta \times \nabla \gamma .
$$

We use (7) to algebraically eliminate the $\mathbf{v}$ in $S$ in favor of the Clebsch potentials $\phi, \beta, \gamma$. This leads to a new action [12]:

$$
S_{\text {new }}=\int \mathrm{d} t \mathrm{~d}^{3} x\left\{\frac{1}{2} \rho(\nabla \phi+\beta \nabla \gamma)^{2}+\rho(\dot{\phi}+\beta \dot{\gamma})+u(\rho)\right\} .
$$

Varying the remaining variables in (9) gives the equations of motion:

$$
\begin{array}{llllll}
\delta \phi: & \dot{\rho}+\nabla \cdot(\rho \mathbf{v})=0, & \delta \beta: & \rho(\dot{\gamma}+(\mathbf{v} \cdot \nabla) \gamma)=0 \quad & \Rightarrow & \dot{\gamma}+(\mathbf{v} \cdot \nabla) \gamma=0, \\
\delta \gamma: & \partial_{t}(\rho \beta)+\nabla(\mathbf{v} \rho \beta)=0 & \Rightarrow & \dot{\beta}+(\mathbf{v} \cdot \nabla) \beta=0, & \delta \rho: & \frac{1}{2} v^{2}+\dot{\phi}+\beta \dot{\gamma}+\mu=0,
\end{array}
$$

where, in the last line, $\mu=\mathrm{d} u / \mathrm{d} \rho$ is the specific enthalpy. We see that the values of both $\beta$ and $\gamma$ are advected with the motion. In Appendix A, we verify that the above equations reproduce Euler's equation.

It is important to realize that the Clebsch decomposition is radically different from the Helmholtz decomposition (Hodge decomposition):

$$
\mathbf{v}=\nabla \Phi+\nabla \times \mathbf{A}
$$

that is more commonly used in electrodynamics and related fields. The Clebsch representation, though less commonly used, is more fundamental when it comes to investigations in fluid dynamics (see for example [17-19]). 


\section{Fluctuations}

We want to study the evolution of small fluctuations superposed on a background flow. We will take the background flow to be described by the set of variables $\left(\rho_{0}, \phi_{0}, \beta_{0}, \gamma_{0}\right)$, and take

$$
\rho=\rho_{0}+\epsilon \rho_{1}, \quad \phi=\phi_{0}+\epsilon \phi_{1}, \quad \beta=\beta_{0}+\epsilon \beta_{1}, \quad \gamma=\gamma_{0}+\epsilon \gamma_{1},
$$

where $\epsilon$ is a (small) dimensionless expansion parameter, to describe the background flow plus perturbation. We will not assume that the background flow is steady, only that it satisfies the equations of motion. We now expand the action $S_{\text {new }}$ out to quadratic order in the fluctuations:

$$
S_{\text {new }}=S_{0}+S_{1}+S_{2}+\cdots .
$$

The action $S_{1}$, containing terms linear in the fluctuations, vanishes because of our assumption that the zeroth order variables obey the equation of motion. The term quadratic in the fluctuations is

$$
S_{2}=\int \mathrm{d} t \mathrm{~d}^{3} x\left\{\frac{1}{2} \rho_{0} \mathbf{v}_{1}^{2}+\rho_{1} \mathbf{v}_{0} \cdot \mathbf{v}_{1}+\rho_{1}\left(\dot{\phi}_{1}+\beta_{0} \dot{\gamma}_{1}+\beta_{1} \dot{\gamma}_{0}\right)+\rho_{0} \beta_{1} \dot{\gamma}_{1}+\frac{1}{2} \frac{c^{2}}{\rho_{0}} \rho_{1}^{2}\right\},
$$

where $\mathbf{v}_{1}$ is shorthand for $\nabla \phi_{1}+\beta_{1} \nabla \gamma_{0}+\beta_{0} \nabla \gamma_{1}$, and

$$
c^{2}=\rho_{0} \frac{\mathrm{d}^{2} u}{\mathrm{~d} \rho^{2}}
$$

is the square of the local speed of sound.

In making this expansion we have ignored the fact that the nonlinearity of the constitutive relations for the fluid, and the nonlinearity of the equation of continuity, mean that Eq. (12) should be supplemented $\mathrm{O}\left(\epsilon^{2}\right)$ corrections, and that these are of the same order as the terms retained in (14). This seeming inconsistency, however, is the usual approximation of linear acoustics: any order $\mathrm{O}\left(\epsilon^{2}\right)$ term in Eq. (12) contributes to $S_{2}$ only linearly, through terms that vanish because the zeroth order variables obey the equation of motion. The omitted terms can be significant at higher order, when computing such effects as radiation stress and mass transport by the sound wave, which are intrinsically of second-order in the wave amplitude, but are unimportant for computing the $\mathrm{O}(\epsilon)$ wave amplitude.

From $S_{2}$ we can deduce the equations of motion for the first-order fluctuating quantities. These equations are not easy to work with, however. Because they are advected with the flow, the potentials $\beta_{0}$ and $\gamma_{0}$ which appear as coefficients in the equations will generally be time-dependent—even if the background flow is steady. Furthermore, there is an overall gauge ambiguity inherent in the Clebsch decomposition which obscures any physical interpretation. (The genesis and nature of this gauge ambiguity is more fully developed in Appendix B.) It is therefore fruitful to seek combinations of the potentials that are gauge-invariant and can be expressed in terms of physical quantities. For example the first-order velocity field:

$$
\mathbf{v}_{1}=\nabla \phi_{1}+\beta_{0} \nabla \gamma_{1}+\beta_{1} \nabla \gamma_{0}
$$

is gauge-invariant because $\mathbf{v}$ is.

By varying $\rho_{1}$ in (14) we find

$$
\rho_{1}=-\frac{\rho_{0}}{c^{2}}\left(\dot{\phi}_{1}+\mathbf{v}_{0} \cdot \nabla \phi_{1}+\beta_{0}\left(\dot{\gamma}_{1}+\mathbf{v}_{0} \cdot \nabla \gamma_{1}\right)+\beta_{1}\left(\dot{\gamma}_{0}+\mathbf{v}_{0} \cdot \nabla \gamma_{0}\right)\right) .
$$

Since

$$
\dot{\beta}_{0}+\mathbf{v}_{0} \cdot \nabla \beta_{0}=0, \quad \dot{\gamma}_{0}+\mathbf{v}_{0} \cdot \nabla \gamma_{0}=0,
$$


we can write this as

$$
\rho_{1}=-\frac{\rho_{0}}{c^{2}} \frac{\mathrm{d} \psi_{1}}{\mathrm{~d} t}
$$

where

$$
\psi_{1}=\phi_{1}+\beta_{0} \gamma_{1}
$$

and

$$
\frac{\mathrm{d}}{\mathrm{d} t}=\frac{\partial}{\partial t}+\mathbf{v}_{0} \cdot \nabla
$$

is the convective derivative.

Note that the density fluctuation $\rho_{1}$, being a physical variable, must be gauge-invariant in the sense of Appendix B. Consequently Eq. (19) suggests that the combination $\psi_{1}$ is itself gauge-invariant. This is easily confirmed. In the notation of Appendix B:

$$
\begin{aligned}
\delta \psi_{1} & =\delta\left(\phi_{1}+\beta_{0} \gamma_{1}\right) \\
& =\left\{\frac{\partial \mathcal{F}}{\partial \beta} \beta_{1}+\frac{\partial \mathcal{F}}{\partial \gamma} \gamma_{1}-\beta_{1} \frac{\partial \mathcal{F}}{\partial \beta}-\beta_{0}\left(\frac{\partial^{2} \mathcal{F}}{\partial \beta^{2}} \beta_{1}+\frac{\partial^{2} \mathcal{F}}{\partial \beta \partial \gamma} \gamma_{1}\right)-\frac{\partial \mathcal{F}}{\partial \gamma} \gamma_{1}+\beta_{0}\left(\frac{\partial^{2} \mathcal{F}}{\partial \beta^{2}} \beta_{1}+\frac{\partial^{2} \mathcal{F}}{\partial \beta \partial \gamma} \gamma_{1}\right)\right\}=0 .
\end{aligned}
$$

We can use $\psi_{1}$ to write

$$
\mathbf{v}_{1}=\nabla \psi_{1}+\xi_{1},
$$

where

$$
\xi_{1}=\beta_{1} \nabla \gamma_{0}-\gamma_{1} \nabla \beta_{0} .
$$

This is a decomposition of the first-order velocity fluctuation into two gauge-invariant parts. Because sound in a fluid is a scalar excitation, it is natural to identify the scalar field $\psi_{1}$ with the acoustic degree of freedom, and $\xi_{1}$, the correction to potential flow induced by angular momentum conservation, with a partial hybridization of the sound with other modes. (Note that there is no requirement that $\nabla \cdot \xi_{1}=0$, which fundamentally distinguishes this procedure from a Helmholtz-type decomposition.) Although the vector field $\xi_{1}$ has three components, it only represents two degrees of freedom. This is because

$$
\xi_{1} \cdot \omega_{0}=\left(\beta_{1} \nabla \gamma_{0}-\gamma_{1} \nabla \beta_{0}\right) \cdot\left(\nabla \beta_{0} \times \nabla \gamma_{0}\right) \equiv 0 .
$$

Since $\xi_{1}$ is gauge-invariant, it should be possible to write it in terms of physical variables. In Section 6 we will show that it is equal to $\mathbf{x}_{1} \times \omega_{0}$ where $\epsilon \mathbf{x}_{1}$ is the particle displacement caused by the disturbance.

\section{Wave equation}

The first-order continuity equation:

$$
\frac{\partial \rho_{1}}{\partial t}+\mathbf{v}_{0} \cdot \nabla \rho_{1}+\rho_{1} \nabla \cdot \mathbf{v}_{0}+\nabla \cdot \rho_{0} \mathbf{v}_{1}=0,
$$

together with the zeroth order continuity equation:

$$
\frac{\partial \rho_{0}}{\partial t}+\nabla \cdot\left(\rho_{0} \mathbf{v}_{0}\right)=0,
$$


the equation for $\rho_{1}$

$$
\rho_{1}=-\frac{\rho_{0}}{c^{2}} \frac{\mathrm{d} \psi_{1}}{\mathrm{~d} t}
$$

and the decomposition $\mathbf{v}_{1}=\nabla \psi_{1}+\xi_{1}$, may be combined to give

$$
\frac{\mathrm{d}}{\mathrm{d} t}\left(\frac{1}{c^{2}} \frac{\mathrm{d}}{\mathrm{d} t} \psi_{1}\right)=\frac{1}{\rho_{0}} \nabla\left(\rho_{0}\left(\nabla \psi_{1}+\xi_{1}\right)\right) .
$$

If we ignore the $\xi_{1},(29)$ is Pierce's approximate wave equation:

$$
\left(\frac{\partial}{\partial t}+\mathbf{v}_{0} \cdot \nabla\right) \frac{1}{c^{2}}\left(\frac{\partial}{\partial t}+\mathbf{v}_{0} \cdot \nabla\right) \psi_{1}=\frac{1}{\rho_{0}} \nabla\left(\rho_{0} \nabla \psi_{1}\right)
$$

(For other approximate wave equations see, for instance [20].) By using Eq. (27) again, this can be rewritten as

$$
\left(\frac{\partial}{\partial t}+\nabla \cdot \mathbf{v}_{0}\right) \frac{\rho_{0}}{c^{2}}\left(\frac{\partial}{\partial t}+\mathbf{v}_{0} \cdot \nabla\right) \psi_{1}=\nabla\left(\rho_{0} \nabla \psi_{1}\right)
$$

where each $\nabla$ is acting on everything to its right. Although (30) may seem more natural, the form (31) has the advantage that it can be written as

$$
\frac{1}{\sqrt{-g}} \partial_{\mu}\left(\sqrt{-g} g^{\mu \nu} \partial_{\nu} \psi_{1}\right)=0
$$

where

$$
\sqrt{-g} g^{\mu \nu}=\frac{\rho_{0}}{c^{2}}\left(\begin{array}{cc}
1 & \mathbf{v}_{0}^{\mathrm{T}} \\
\mathbf{v}_{0} & \mathbf{v}_{0} \mathbf{v}_{0}^{\mathrm{T}}-c^{2} \mathbf{I}
\end{array}\right) .
$$

We use the convention that Greek letters run over four space-time indices $0,1,2,3$ with $0 \equiv t$, while Roman indices refer to the three space components. Eq. (32) has the same form as that of a scalar wave propagating in a gravitational field with pseudo-Riemann (Lorentzian) metric $g_{\mu \nu}$. We will refer to $g_{\mu \nu}$ as the acoustic metric. The idea of writing the sound wave equation in this way is due to $[2,3]$.

As is customary in general relativity, the symbol $g$ denotes the determinant of the covariant form of the metric, $g_{\mu \nu}$, so det $g^{\mu \nu}=g^{-1}$. Taking the determinant of both sides of (33) thus shows that the 4-volume measure $\sqrt{-g}$ is equal to $\rho_{0}^{2} / c$. Knowing this, we may then invert the matrix $g^{\mu v}$ to find the covariant components of the metric:

$$
g_{\mu \nu}=\frac{\rho_{0}}{c}\left(\begin{array}{cc}
c^{2}-v_{0}^{2} & \mathbf{v}_{0}^{\mathrm{T}} \\
\mathbf{v}_{0} & -\mathbf{I}
\end{array}\right)
$$

The associated space-time interval is therefore

$$
\mathrm{d} s^{2}=\frac{\rho_{0}}{c}\left\{c^{2} \mathrm{~d} t^{2}-\delta_{i j}\left(\mathrm{~d} x^{i}-v_{0}^{i} \mathrm{~d} t\right)\left(\mathrm{d} x^{j}-v_{0}^{j} \mathrm{~d} t\right)\right\} .
$$

In the geometric acoustics limit, sound propagates along the null geodesics of this metric.

Metrics of the form (35), although without the overall conformal factor $\rho_{0} / c$, appear in the Arnowitt-Deser-Misner (ADM) formalism of general relativity [22]. There, $c$ and $-v_{0}^{i}$ are referred to as the lapse function and shift vector, respectively. They serve to glue successive three-dimensional time slices together to form a four-dimensional space-time; for a picture see [23]. In our present case, provided $\rho_{0} / c$ can be regarded as a constant, each 3-space is ordinary flat $\mathbf{R}^{3}$ equipped with the rectangular Cartesian metric $g_{i j}^{\text {(space) }}=\delta_{i j}$-but the resultant space-time is in general curved, the curvature depending on the degree of inhomogeneity of the mean flow $\mathbf{v}_{0}$. 
This formalism is very pretty, but (30) is exact only when the background flow is potential. Eq. (29), on the other hand, is valid for a general barotropic flow_but to be of use it must be complemented by an equation determining the time evolution of $\xi_{1}$. We now derive such an equation. We start with the observation that, since $\beta, \gamma$ are convectively conserved, we have

$$
\frac{\partial \beta_{0}}{\partial t}+\left(\mathbf{v}_{0} \cdot \nabla\right) \beta_{0}=0
$$

and

$$
\frac{\partial \beta_{1}}{\partial t}+\left(\mathbf{v}_{0} \cdot \nabla\right) \beta_{1}+\left(\mathbf{v}_{1} \cdot \nabla\right) \beta_{0}=0
$$

Taking the gradient of (36) gives

$$
\left(\frac{\partial}{\partial t}+\mathbf{v}_{0} \cdot \nabla\right) \nabla_{i} \beta_{0}=-\left(\nabla_{i} v_{0 j}\right) \nabla_{j} \beta_{0} .
$$

Thus, using the definition (24):

$$
\begin{aligned}
\left(\frac{\partial}{\partial t}+\mathbf{v}_{0} \cdot \nabla\right) \xi_{1 i} & =-\left[\left(\mathbf{v}_{1} \cdot \nabla\right) \beta_{0}\right] \nabla_{i} \gamma_{0}+\left[\left(\mathbf{v}_{1} \cdot \nabla\right) \gamma_{0}\right] \nabla_{i} \beta_{0}-\beta_{1}\left(\nabla_{i} v_{0 j}\right) \nabla_{j} \gamma_{0}+\gamma_{1}\left(\nabla_{i} v_{0 j}\right) \nabla_{j} \beta_{0} \\
& =-v_{1 j}\left(\nabla_{j} \beta_{0} \nabla_{i} \gamma_{0}-\nabla_{j} \gamma_{0} \nabla_{i} \beta_{0}\right)-\left(\nabla_{i} v_{0 j}\right) \xi_{1 j}=-v_{1 j}\left(\nabla_{j} v_{0 i}-\nabla_{i} v_{0 j}\right)-\left(\nabla_{i} v_{0 j}\right) \xi_{1 j} \\
& =\left(-\nabla_{j} \psi_{1}-\xi_{1 j}\right)\left(\nabla_{j} v_{0 i}-\nabla_{i} v_{0 j}\right)-\left(\nabla_{i} v_{0 j}\right) \xi_{1 j}=-\nabla_{j} \psi_{1}\left(\nabla_{j} v_{0 i}-\nabla_{i} v_{0 j}\right)-\xi_{1 j}\left(\nabla_{j} v_{0 i}\right)
\end{aligned}
$$

which can be written as

$$
\frac{\mathrm{d} \xi_{1}}{\mathrm{~d} t}=\nabla \psi_{1} \times \omega_{0}-\left(\xi_{1} \cdot \nabla\right) \mathbf{v}_{0}
$$

In summary: the two coupled equations:

$$
\frac{\mathrm{d}}{\mathrm{d} t}\left(\frac{1}{c^{2}} \frac{\mathrm{d}}{\mathrm{d} t} \psi_{1}\right)=\frac{1}{\rho_{0}} \nabla\left(\rho_{0}\left(\nabla \psi_{1}+\xi_{1}\right)\right)
$$

and

$$
\frac{\mathrm{d} \xi_{1}}{\mathrm{~d} t}=\nabla \psi_{1} \times \omega_{0}-\left(\xi_{1} \cdot \nabla\right) \mathbf{v}_{0}
$$

form a complete exact closed system of equations, containing only gauge-invariant quantities, describing the first-order fluctuations about the background mean flow.

\section{Displacement field}

It is not yet clear that, under most circumstances of interest in acoustics, the quantity $\xi_{1}$ is a small correction to $\nabla \psi_{1}$. It becomes so, however, once we establish the result

$$
\xi_{1}=\mathbf{x}_{1} \times \omega_{0},
$$

where $\epsilon \mathbf{x}_{1}$ is the displacement of a material particle due to the sound wave. By "displacement" we mean that the material point which in the unperturbed reference flow was at time $t$ located at $\mathbf{x}$ is, as a result of the perturbation, now to be found at position $\mathbf{x}+\epsilon \mathbf{x}_{1}$. Given (43), we see that the order of magnitude of $\xi_{1}$ is that of the product of 
the displacement amplitude with the background flow rotation frequency. The fluctuating velocity associated with the acoustic field is, on the other hand, of the order of the displacement amplitude times the frequency, $\Omega$, of the sound wave. Thus $\xi_{1}$ is smaller than $\nabla \psi_{1}$ by a factor of $\left|\omega_{0}\right| / \Omega$.

Observe that this argument tacitly assumes that $\mathbf{x}_{1}$ remains small and oscillating. This is certainly what we expect for a sound wave, but, in the absence of viscous damping, many flows with vorticity will be unstable to the onset of turbulence, and if the sound triggers such an instability $\mathbf{x}_{1}$ will grow without bound. In this case, the entire notion of sound propagating in an unperturbed background flow becomes meaningless. Our equations will continue to be valid in the initial stages of this growth, however, and so they may be of value in investigating the stability of flows against the onset of turbulence.

To establish (43) we recall that $\mathbf{x}_{1}(\mathbf{x}, t)$ was defined by taking the material point that was at time $t$ located at $\mathbf{x}$, is, as a result of the perturbation, now to be found at $\mathbf{x}+\epsilon \mathbf{x}_{1}$. We also remember that the numerical values of the potentials $\beta, \gamma$, are painted on the material particles, and so move with the flow under both time evolution and the creation of an initial perturbation by means of an external potential body force. Interpreting this statement mathematically leads to

$$
\mathbf{x}_{1} \cdot \nabla \beta_{0}+\beta_{1}=0, \quad \mathbf{x}_{1} \cdot \nabla \gamma_{0}+\gamma_{1}=0 .
$$

From this we may write

$$
\beta_{1} \nabla \gamma_{0}-\gamma_{1} \nabla \beta_{0}=\left(\mathbf{x}_{1} \cdot \nabla \gamma_{0}\right) \nabla \beta_{0}-\left(\mathbf{x}_{1} \cdot \nabla \beta_{0}\right) \nabla \gamma_{0},=\mathbf{x}_{1} \times\left(\nabla \beta_{0} \times \nabla \gamma_{0}\right),=\mathbf{x}_{1} \times \omega_{0} .
$$

We can use (43) to re-derive the equation of motion for $\xi_{1}$ and so provide a derivation of the wave equation that is independent of the use of Clebsch potentials. In their absence, though, the origin of the decomposition of the velocity field into the sum of $\xi_{1}=\mathbf{x}_{1} \times \omega_{0}$ and the gradient of the velocity potential, $\psi_{1}$, is more than a trifle obscure.

To verify that (43) leads to the equation of motion (39) for $\xi_{1}$ we must first establish a connection between $\mathbf{v}_{1}$ and the time derivative of $\mathbf{x}_{1}$. This requires us to describe the perturbation with a little more formality. Consider a family $\mathbf{v}(\mathbf{x}, t, \lambda)$ of adjacent solutions of the full equations of motion. The velocity field $\mathbf{v}(\mathbf{x}, t, 0)$ is that of the unperturbed reference flow, and increasing values of $\lambda$ correspond to flows evolving from a one-parameter family of initial perturbations. By definition the operations of time evolution and variation of $\lambda$ commute.

The position, $\mathbf{x}(t, \lambda)$, of a material particle is given by the solution to the differential equation:

$$
\dot{\mathbf{x}}(t)=\mathbf{v}(\mathbf{x}(t, \lambda), t, \lambda)
$$

with suitable initial conditions. Our first-order perturbed fields are, in this language:

$$
\mathbf{x}_{1}=\left.\frac{\mathrm{d} \mathbf{x}}{\mathrm{d} \lambda}\right|_{\lambda=0}, \quad \mathbf{v}_{1}=\left.\frac{\mathrm{d} \mathbf{v}}{\mathrm{d} \lambda}\right|_{\lambda=0} .
$$

Differentiating (46) with respect to $\lambda$, and interpreting the time derivative as a convective derivative, gives

$$
\mathbf{v}_{1}=\frac{\partial \mathbf{x}_{1}}{\partial t}+\left(\mathbf{v}_{0} \cdot \nabla\right) \mathbf{x}_{1}-\left(\mathbf{x}_{1} \cdot \nabla\right) \mathbf{v}_{0} .
$$

Now, starting from

$$
\xi_{1}=\mathbf{x}_{1} \times \omega_{0}
$$

and the convective derivatives

$$
\begin{aligned}
\frac{\mathrm{d} \mathbf{x}_{1}}{\mathrm{~d} t} & =\mathbf{v}_{1}+\left(\mathbf{x}_{1} \cdot \nabla\right) \mathbf{v}_{0}, \\
\frac{\mathrm{d} \omega_{0}}{\mathrm{~d} t} & =\left(\omega_{0} \cdot \nabla\right) \mathbf{v}_{0}-\left(\nabla \cdot \mathbf{v}_{0}\right) \omega_{0},
\end{aligned}
$$


we may find an equation for the time evolution of $\xi_{1}$. Using the fact the convective derivative is a derivation, we find

$$
\begin{aligned}
\frac{\mathrm{d} \xi_{1}}{\mathrm{~d} t} & =\left(\mathbf{v}_{1}+\left(\mathbf{x}_{1} \cdot \nabla\right) \mathbf{v}_{0}\right) \times \omega_{0}+\mathbf{x}_{1} \times\left(\left(\omega_{0} \cdot \nabla\right) \mathbf{v}_{0}-\left(\nabla \cdot \mathbf{v}_{0}\right) \omega_{0}\right) \\
& =\mathbf{v}_{1} \times \omega_{0}+\left(\nabla \cdot \mathbf{x}_{1}\right)\left(\mathbf{v}_{0} \times \omega_{0}\right)+\left(\nabla \cdot \omega_{0}\right)\left(\mathbf{x}_{1} \times \mathbf{v}_{0}\right)+\left(\nabla \cdot \mathbf{v}_{0}\right)\left(\omega_{0} \times \mathbf{x}_{1}\right) .
\end{aligned}
$$

In the second line the ordering of the symbols is meant only to indicate how the indices are wired up. The $\nabla$ must be understood to act to the right only on the velocity field $\mathbf{v}_{0}$.

We now use the vector identity:

$$
(\mathbf{x} \cdot \mathbf{a})(\mathbf{b} \times \mathbf{c})+(\mathbf{x} \cdot \mathbf{b})(\mathbf{c} \times \mathbf{a})+(\mathbf{x} \cdot \mathbf{c})(\mathbf{a} \times \mathbf{b})=\mathbf{x}[\mathbf{a} \cdot(\mathbf{b} \times \mathbf{c})]
$$

with $\mathbf{x}$ replaced by $\nabla$ (still acting only on $\mathbf{v}_{0}$ ) to find that

$$
\begin{aligned}
\frac{\mathrm{d} \xi_{1}}{\mathrm{~d} t} & =\mathbf{v}_{1} \times \omega_{0}+\nabla\left(\mathbf{x}_{1} \cdot\left(\mathbf{v}_{0} \times \omega_{0}\right)\right)=\mathbf{v}_{1} \times \omega_{0}-\nabla\left(\mathbf{v}_{0} \cdot\left(\mathbf{x}_{1} \times \omega_{0}\right)\right) \\
& =\mathbf{v}_{1} \times \omega_{0}-\nabla\left(\mathbf{v}_{0} \cdot \xi_{1}\right)=\left(\nabla \psi_{1}\right) \times \omega_{0}-\left(\xi_{1} \cdot \nabla\right) \mathbf{v}_{0}
\end{aligned}
$$

which is the same as (40). (Again, in the first three lines, $\nabla$ must be understood to act only on $\mathbf{v}_{0}$, even though it may be written to the left of other variables.)

We can also check the consistency of the time evolution of the first-order vorticity. From (45) we find that

$$
\omega_{1}=\nabla \times\left(\mathbf{x}_{1} \times \omega_{0}\right),
$$

so

$$
\frac{\partial \omega_{1}}{\partial t}=\nabla \times\left(\frac{\partial \mathbf{x}_{1}}{\partial t} \times \omega_{0}\right)+\nabla \times\left(\mathbf{x}_{1} \times \frac{\partial \omega_{0}}{\partial t}\right) .
$$

It is not immediately obvious that (56) is compatible with the equation:

$$
\frac{\partial \omega_{1}}{\partial t}=\nabla \times\left(\mathbf{v}_{0} \times \omega_{1}\right)+\nabla \times\left(\mathbf{v}_{1} \times \omega_{0}\right)
$$

which comes from applying $\mathrm{d} / \mathrm{d} \lambda$ to the vorticity evolution equation:

$$
\frac{\partial \omega}{\partial t}=\nabla \times(\mathbf{v} \times \omega) .
$$

The right-hand sides of (56) and (57) are equal only if

$$
\frac{\partial \mathbf{x}_{1}}{\partial t} \times \omega_{0}+\mathbf{x}_{1} \times \frac{\partial \omega_{0}}{\partial t}-\mathbf{v}_{0} \times \omega_{1}-\mathbf{v}_{1} \times \omega_{0}
$$

is the gradient of something. Now by using (48), (55) and (57), we can write (59) as

$$
\begin{gathered}
\omega_{0} \times\left(\nabla \times\left(\mathbf{x}_{1} \times \mathbf{v}_{0}\right)\right)+\mathbf{x}_{1} \times\left(\nabla \times\left(\mathbf{v}_{0} \times \omega_{0}\right)\right)+\mathbf{v}_{0} \times\left(\nabla \times\left(\omega_{0} \times \mathbf{x}_{1}\right)\right) \\
-\left(\omega_{0} \times \mathbf{x}_{1}\right)\left(\nabla \cdot \mathbf{v}_{0}\right)-\left(\mathbf{x}_{1} \times \mathbf{v}_{0}\right)\left(\nabla \cdot \omega_{0}\right)-\left(\mathbf{v}_{0} \times \omega_{0}\right)\left(\nabla \cdot \mathbf{x}_{1}\right) .
\end{gathered}
$$

Here we have added in a term $\left(\mathbf{x}_{1} \times \mathbf{v}_{0}\right)\left(\nabla \cdot \omega_{0}\right)$, which is of course identically zero, in order to preserve manifest cyclic symmetry of the terms.

Now for any three vector fields $\mathbf{a}, \mathbf{b}, \mathbf{c}$, we may verify that

$$
\begin{aligned}
\mathbf{a} \times(\nabla \times(\mathbf{b} \times \mathbf{c}))+\mathbf{b} \times(\nabla \times(\mathbf{c} \times \mathbf{a}))+\mathbf{c} \times(\nabla \times(\mathbf{a} \times \mathbf{b})) \\
\quad-(\mathbf{a} \times \mathbf{b})(\nabla \cdot \mathbf{c})-(\mathbf{b} \times \mathbf{c})(\nabla \cdot \mathbf{a})-(\mathbf{c} \times \mathbf{a})(\nabla \cdot \mathbf{b})=\nabla(\mathbf{a} \cdot(\mathbf{b} \times \mathbf{c})),
\end{aligned}
$$

where $\nabla$ is acting on everything to its right. Applying this to (60) shows that it is a total derivative, and so the evolution equations are consistent. 


\section{Illustrative examples}

As illustrative examples of the formalism consider waves propagating in the background flow

$$
\mathbf{v}_{0}=\frac{\omega_{0}}{2}\left(\begin{array}{c}
-y \\
x \\
0
\end{array}\right)
$$

This corresponds to the fluid rotating as a rigid body with angular frequency $\omega_{0} / 2$. The perverse notation for the frequency arises because we have been using the symbol $\omega$ to denote vorticity, and $\nabla \times \mathbf{v}_{0}=\omega_{0} \hat{\mathbf{z}}$. (A more traditional discussion of this example my be found, for instance, in [24].) To reduce notational clutter, in this section we will drop the suffix 1 from the fields $\psi$ and $\xi$. It should still be borne in mind that they are first-order quantities.

\subsection{Sound/inertial-wave hybridization}

Our equations of motion are

$$
-\frac{\mathrm{d}}{\mathrm{d} t}\left(\frac{1}{c^{2}} \frac{\mathrm{d} \psi}{\mathrm{d} t}\right)+\frac{1}{\rho_{0}} \nabla\left(\rho_{0}(\nabla \psi+\xi)\right)=0, \quad \frac{\mathrm{d} \xi}{\mathrm{d} t}-\left(\nabla \psi \times \omega_{0}\right)+(\xi \cdot \nabla) \mathbf{v}_{0}=0 .
$$

They need to be supplemented with an initial condition that sets $\xi \cdot \omega_{0}=0$. This orthogonality is then preserved by the subsequent motion. We will ignore any effects due to gradients in $\rho_{0}$ and $c^{2}$.

Take as ansatz a plane wave in the frame rotating with the fluid:

$$
\xi=\left(\xi_{x^{\prime}} \hat{\mathbf{x}}^{\prime}+\xi_{y^{\prime}} \hat{\mathbf{y}}^{\prime}\right)=\left(\Xi_{x^{\prime}} \hat{\mathbf{x}}^{\prime}+\Xi_{y^{\prime}} \hat{\mathbf{y}}^{\prime}\right) \mathrm{e}^{\mathrm{i}\left(k_{x^{\prime}} x^{\prime}+k_{y^{\prime}} y^{\prime}+k_{z^{\prime}} z^{\prime}-\Omega t\right)}, \quad \psi=\Psi \mathrm{e}^{\mathrm{i}\left(k_{x^{\prime}} x^{\prime}+k_{y^{\prime}} y^{\prime}+k_{z^{\prime}} z^{\prime}-\Omega t\right)} .
$$

Here $\Xi_{x^{\prime}, y^{\prime}}$ and $\Psi$ are constant amplitudes. The primed unit vectors are

$$
\hat{\mathbf{x}}^{\prime}=\hat{\mathbf{x}} \cos \left[\left(\frac{1}{2} \omega_{0}\right) t\right]+\hat{\mathbf{y}} \sin \left[\left(\frac{1}{2} \omega_{0}\right) t\right], \quad \hat{\mathbf{y}}^{\prime}=-\hat{\mathbf{x}} \sin \left[\left(\frac{1}{2} \omega_{0}\right) t\right]+\hat{\mathbf{y}} \cos \left[\left(\frac{1}{2} \omega_{0}\right) t\right], \quad \hat{\mathbf{z}}^{\prime}=\hat{\mathbf{z}}
$$

and the primed co-ordinates

$$
x^{\prime}=x \cos \left[\left(\frac{1}{2} \omega_{0}\right) t\right]+y \sin \left[\left(\frac{1}{2} \omega_{0}\right) t\right], \quad y^{\prime}=-x \sin \left[\left(\frac{1}{2} \omega_{0}\right) t\right]+y \cos \left[\left(\frac{1}{2} \omega_{0}\right) t\right], \quad z^{\prime}=z .
$$

The convective derivatives on $\psi$ and on the components of $\xi$ become

$$
\frac{\mathrm{d} \psi}{\mathrm{d} t}=\left(\frac{\partial \psi}{\partial t}\right)_{x^{\prime}, y^{\prime}}=-\mathrm{i} \Omega \psi, \quad \frac{\mathrm{d} \xi_{x^{\prime}, y^{\prime}}}{\mathrm{d} t}=\left(\frac{\partial \xi_{x^{\prime}, y^{\prime}}}{\partial t}\right)_{x^{\prime}, y^{\prime}}=-\mathrm{i} \Omega \xi_{x^{\prime}, y^{\prime}} .
$$

For $\xi$ itself we need to take note of the time dependence of the unit vectors $\hat{\mathbf{x}}^{\prime}, \hat{\mathbf{y}}^{\prime}$, so we have

$$
\begin{aligned}
\frac{\mathrm{d} \xi}{\mathrm{d} t} & =\left(\frac{\mathrm{d} \xi_{x^{\prime}}}{\mathrm{d} t}-\left(\frac{\omega_{0}}{2}\right) \xi_{y^{\prime}}\right) \hat{\mathbf{x}}^{\prime}+\left(\frac{\mathrm{d} \xi_{y^{\prime}}}{\mathrm{d} t}+\left(\frac{\omega_{0}}{2}\right) \xi_{x^{\prime}}\right) \hat{\mathbf{y}}^{\prime} \\
& =\left(-\mathrm{i} \Omega \xi_{x^{\prime}}-\left(\frac{\omega_{0}}{2}\right) \xi_{y^{\prime}}\right) \hat{\mathbf{x}}^{\prime}+\left(-\mathrm{i} \Omega \xi_{y^{\prime}}+\left(\frac{\omega_{0}}{2}\right) \xi_{x^{\prime}}\right) \hat{\mathbf{y}}^{\prime} .
\end{aligned}
$$

Also we need

$$
(\xi \cdot \nabla) \mathbf{v}_{0}=-\xi_{y^{\prime}}\left(\frac{1}{2} \omega_{0}\right) \hat{\mathbf{x}}^{\prime}+\xi_{y^{\prime}}\left(\frac{1}{2} \omega_{0}\right) \hat{\mathbf{y}}^{\prime} .
$$

The two off-diagonal $\omega_{0} / 2$ terms add to get rid of the $1 / 2$. The coupled equations therefore become

$$
\left(\begin{array}{ccc}
-\mathrm{i} \Omega & -\omega_{0} & -\mathrm{i} k_{y^{\prime}} \omega_{0} \\
+\omega_{0} & -\mathrm{i} \Omega & +\mathrm{i} k_{x^{\prime}} \omega_{0} \\
+\mathrm{i} k_{x^{\prime}} & \mathrm{i} k_{y^{\prime}} & \left(\frac{\Omega^{2}}{c^{2}}-k^{2}\right)
\end{array}\right)\left(\begin{array}{c}
\Xi_{x^{\prime}} \\
\Xi_{y^{\prime}} \\
\Psi
\end{array}\right)=0 .
$$


For a solution to exist, the determinant of the matrix in (70) must vanish. This gives the dispersion relation:

$$
\left(\omega_{0}^{2}-\Omega^{2}\right)\left(\frac{\Omega^{2}}{c^{2}}-|k|^{2}\right)+\omega_{0}^{2} k_{x^{\prime}}^{2}+\omega_{0}^{2} k_{y^{\prime}}^{2}=0
$$

which for fixed $\mathbf{k}$ is a quadratic equation for $\Omega^{2}$.

Some insight into this dispersion relation can be obtained by letting $c^{2} \rightarrow \infty$. In this limit the quadratic reduces to

$$
|k|^{2} \Omega^{2}-\omega_{0}^{2} k_{z^{\prime}}^{2}=0
$$

and so gives

$$
\Omega^{2}=\frac{\omega_{0}^{2} k_{z^{\prime}}^{2}}{k_{x^{\prime}}^{2}+k_{y^{\prime}}^{2}+k_{z^{\prime}}^{2}} .
$$

This is the well-known dispersion relation for inertial waves in an incompressible fluid [25]. For these modes the restoring force comes entirely from angular momentum conservation. They are low frequency, $\Omega^{2} \leq \omega_{0}^{2}$, oscillations and have a number of unusual features. In particular the frequency is independent of the magnitude of $\mathbf{k}$, so the group velocity is perpendicular to the phase velocity-i.e. parallel to the wavecrests. At any particular frequency the disturbance spreads out from its source along a diabolic cone.

The second root of the quadratic equation, $\Omega^{2} \approx c^{2} k^{2}$ corresponds to conventional sound, and is lost to infinity as $c^{2}$ becomes large.

Now let us consider general values of $c^{2}$. From the eigenmode equation we can solve for $\xi$ in terms of the amplitude of $\psi$ to get

$$
\left(\begin{array}{c}
\Xi_{x^{\prime}} \\
\Xi_{y^{\prime}}
\end{array}\right)=\frac{\omega_{0}}{\Omega^{2}-\omega_{0}^{2}}\left(\begin{array}{c}
-k_{y^{\prime}} \Omega+\mathrm{i} k_{x^{\prime}} \omega_{0} \\
k_{x^{\prime}} \Omega+\mathrm{i} k_{y^{\prime}} \omega_{0}
\end{array}\right) \Psi .
$$

This appears to be singular when $\Omega^{2}$ approaches $\omega_{0}^{2}$, but, as we will see, this occurs only near $k_{x^{\prime}}=k_{y^{\prime}}=0$ and the limit is smooth, the fluid rotating in circles in the $x-y$ plane.

From $\xi$ we can find the velocity field, $\mathbf{v}_{1}$, and hence, by integration, the first-order displacement field, $\mathbf{x}_{1}$, in the frame rotating with the background fluid. (If $\mathbf{x}_{1}=x_{1 x^{\prime}} \hat{\mathbf{x}}^{\prime}+x_{1 y^{\prime}} \hat{\mathbf{y}}^{\prime}$, and $\mathbf{v}_{1}=v_{1 x^{\prime}} \hat{\mathbf{x}}^{\prime}+v_{1 y^{\prime}} \hat{\mathbf{y}}^{\prime}$, then (48) reduces to $v_{1 x^{\prime}, y^{\prime}}=\left(\partial x_{1 x^{\prime}, y^{\prime}} / \partial t\right)_{x^{\prime}, y^{\prime}}$.) We therefore find

$$
\mathbf{x}_{1}=\left(\frac{\Psi}{\Omega}\right)\left[\left(\begin{array}{c}
-k_{x^{\prime}} \\
-k_{y^{\prime}} \\
-k_{z^{\prime}}
\end{array}\right)+\frac{\omega_{0}}{\Omega^{2}-\omega_{0}^{2}}\left(\begin{array}{c}
-k_{x^{\prime}} \omega_{0}-\mathrm{i} k_{y^{\prime}} \Omega \\
-k_{y^{\prime}} \omega_{0}+\mathrm{i} k_{x^{\prime}} \Omega \\
0
\end{array}\right)\right] \mathrm{e}^{\mathrm{i}\left(k_{x^{\prime}} x^{\prime}+k_{y^{\prime}} y^{\prime}+k_{z^{\prime}} z^{\prime}-\Omega t\right)} .
$$

It is now straightforward to verify that we recover $\xi$ from $\xi=\left(\mathbf{x}_{1} \times \omega_{0}\right)$. We also verify that the correction to potential flow is $\mathrm{O}\left(\omega_{0} / \Omega\right)$ when $\Omega \gg \omega_{0}$.

\subsection{Poincaré waves}

If we restrict ourselves waves with $k_{z}=0$, then setting the determinant to zero gives

$$
\frac{\Omega^{2}}{c^{2}}\left(\omega_{0}^{2}+c^{2} k^{2}-\Omega^{2}\right)=0 .
$$


We therefore have two classes of modes: those with zero frequency, and those with a gapped dispersion relation:

$$
\Omega^{2}=\omega_{0}^{2}+c^{2}\left(k_{x^{\prime}}^{2}+k_{y^{\prime}}^{2}\right) .
$$

The former are $z$-independent geostrophic flows (Taylor-column flows [26,27]) where pressure gradients are balanced against a Coriolis force. The gapped modes are the Poincaré waves [28].

We can obtain the Poincaré modes by considering the motion directly in the $x^{\prime}, y^{\prime}$ frame. The effect of the frame rotation produces a Coriolis force, and so the equation of motion is

$$
\frac{\partial v_{1 x^{\prime}}}{\partial t}=+\omega_{0} v_{1 y^{\prime}}-\frac{c^{2} \nabla_{x^{\prime}} \rho_{1}}{\rho_{0}}, \quad \frac{\partial v_{1 y^{\prime}}}{\partial t}=-\omega_{0} v_{1 x^{\prime}}-\frac{c^{2} \nabla_{y^{\prime}} \rho_{1}}{\rho_{0}} .
$$

To solve we need to combine this with the continuity equation:

$$
\frac{\partial \rho_{1}}{\partial t}+\rho_{0}\left(\nabla_{x^{\prime}} v_{1 x^{\prime}}+\nabla_{y^{\prime}} v_{1 y^{\prime}}\right)=0
$$

For waves travelling in the $\hat{\mathbf{x}}^{\prime}$ direction we find

$$
v_{1 x^{\prime}}=A \cos \left(k x^{\prime}-\Omega t\right), \quad v_{1 y^{\prime}}=A\left(\frac{\omega_{0}}{\Omega}\right) \sin \left(k x^{\prime}-\Omega t\right), \quad \rho_{1}=A\left(\frac{\rho_{0} k}{\Omega}\right) \cos \left(k x^{\prime}-\Omega t\right),
$$

together with the dispersion relation (77). We also find the displacements of the particles to be

$$
x_{1 x^{\prime}}=-A\left(\frac{1}{\Omega}\right) \sin \left(k x^{\prime}-\Omega t\right), \quad x_{1 y^{\prime}}=A\left(\frac{\omega_{0}}{\Omega^{2}}\right) \cos \left(k x^{\prime}-\Omega t\right) .
$$

When $\mathbf{k}=0$, we have $\Omega=\omega_{0}$, and the particles move in circles in the $x, y$ plane. This limiting motion is solenoidal and coincides with the $k_{x^{\prime}}=k_{y^{\prime}}=0$ limit of the incompressible fluid inertial waves.

We may now make contact with our $\psi, \xi$ formalism by writing

$$
\rho_{1}=-\frac{\rho_{0}}{c^{2}} \frac{\mathrm{d} \psi}{\mathrm{d} t}=\frac{\rho_{0}}{c^{2}}\left(\frac{\partial \psi}{\partial t}\right)_{x^{\prime}, y^{\prime}},
$$

where

$$
\psi=A\left(\frac{c^{2} k}{\Omega^{2}}\right) \sin \left(k x^{\prime}-\Omega t\right) .
$$

The relation $\xi=\mathbf{x}_{1} \times \omega_{0}$, which was derived in the $x, y$ inertial frame, continues to hold in the rotating frame without modification. So we have

$$
\xi=\mathbf{x}_{1} \times \omega_{0}=\omega_{0 z}\left(x_{1 y^{\prime}} \hat{\mathbf{x}}^{\prime}-x_{1 x^{\prime}} \hat{\mathbf{y}}^{\prime}\right) .
$$

We can combine this with our expression for $\psi$ to get

$$
v_{1 x^{\prime}}=\nabla_{x^{\prime}} \psi+\xi_{x^{\prime}}=A\left(\frac{c^{2} k^{2}}{\Omega^{2}}+\frac{\omega_{0}^{2}}{\Omega^{2}}\right) \cos \left(k x^{\prime}-\Omega t\right), \quad v_{1 y^{\prime}}=0+\xi_{y^{\prime}}=A\left(\frac{\omega_{0}}{\Omega}\right) \sin \left(k x^{\prime}-\Omega t\right) .
$$

Since the factor in parenthesis in the first line is seen to be unity by use of the dispersion relation, we recover the earlier expression for $\mathbf{v}_{1}$, and confirm that the gauge-invariant decomposition works as advertised. Again we see that the velocity field $\xi$, which arises from angular momentum conservation, is smaller than the pressure induced flow, $\nabla \psi$, by a factor of $\omega_{0} / \Omega$. 


\section{Discussion}

The central idea in this paper is the decomposition $\mathbf{v}_{1}=\nabla \psi+\xi$ of a general velocity perturbation into a potential flow and a correction required by angular momentum conservation. This decomposition is motivated by the Clebsch formalism, but does not depend on it. From the decomposition we see that corrections to the acoustic metric equation depend only on the ratio of the frequency of the sound wave to the frequency, $\omega_{0} / 2$, of the background fluid rotation. This rotation frequency is determined by the antisymmetric part, $\left(\partial_{i} v_{j}-\partial_{j} v_{i}\right) / 2$, of the velocity inhomogeneity. The symmetric part, the rate of strain $e_{i j}=\left(\partial_{i} v_{j}+\partial_{j} v_{i}\right) / 2$, can be large and the correction remain small. This is not unreasonable because the acoustic metric equation is exact for any potential background flow-no matter how inhomogeneous.

At low frequencies the correction $\xi=\mathbf{x}_{1} \times \omega_{0}$ ceases to be negligible. In this regime, the sound waves hybridize with whichever of the many other modes available to a fluid with vorticity happen to have comparable frequency. The hybridization may lead to a spectral gap, as with the Poincaré waves, to birefringence, and to other phenomena which show that the acoustic metric is no longer all that is needed to describe sound propagation.

\section{Acknowledgements}

MS was supported by the NSF (USA) under grant DMR-98-17941. SEPB was supported by FAPERj (Brazil). MV was initially supported by the US Department of Energy, and subsequently by the Marsden Fund (administered by the Royal Society of New Zealand, RSNZ). KEH was supported by the Ministerio de Educacion y Cultura, España, and subsequently by the Fundação para a Ciência e a Tecnologia, Portugal. This work was begun during the workshop Analog models of General Relativity at CBPF, the Brazilian Center for Research in Physics, located in Urca, Rio de Janeiro, Brazil. MV and MS thank the center for their hospitality and for hosting the workshop.

\section{Appendix A. Euler equation}

We now demonstrate that the equations of motion for the Clebsch potentials imply the Euler equation for the fluid. Apply $\nabla_{i}$ to the last line in (10) and add and subtract $\dot{\beta} \nabla_{i} \gamma$, so that the second term is the time derivative of the velocity:

$$
v_{k} \nabla_{i} v_{k}+\partial_{t}\left(\nabla_{i} \phi+\beta \nabla_{i} \gamma\right)-\dot{\beta} \nabla_{i} \gamma+\dot{\gamma} \nabla_{i} \beta=-\nabla_{i} \mu .
$$

In other words

$$
\partial_{t} v_{i}+v_{k} \nabla_{i} v_{k}-\dot{\beta} \nabla_{i} \gamma+\dot{\gamma} \nabla_{i} \beta=-\nabla_{i} \mu .
$$

The second, third, and fourth terms on the left-hand side now need to be taken care of. Write

$$
v_{k} \nabla_{i} v_{k}=v_{k} \nabla_{k} v_{i}+v_{k}\left(\nabla_{i} v_{k}-\nabla_{k} v_{i}\right),=(\mathbf{v} \cdot \nabla) v_{i}+v_{k}\left(\nabla_{i} \beta \nabla_{k} \gamma-\nabla_{k} \beta \nabla_{i} \gamma\right),=(\mathbf{v} \cdot \nabla) v_{i}-\dot{\gamma} \nabla_{i} \beta+\dot{\beta} \nabla_{i} \gamma,
$$

where, in the last line, we have used the convective constancy of $\beta, \gamma$. Inserting (A.3) into (A.2) we find

$$
\dot{\mathbf{v}}+(\mathbf{v} \cdot \nabla) \mathbf{v}=-\nabla \mu
$$

which is Euler's equation. 


\section{Appendix B. Gauge transformations}

In three dimensions any vector field may be locally represented in the form $[15,16]$ :

$$
\mathbf{v}=\nabla \phi+\beta \nabla \gamma
$$

Given a velocity field $\mathbf{v}$, however, the potentials $\phi, \beta$ and $\gamma$, are not uniquely determined. This indeterminacy is usually described as being due to a gauge invariance, but it is more analogous to the residual freedom to make time-independent gauge transformations that survives after we fix the $A_{0}=0$ gauge in Maxwell electrodynamics. As in that example, once we have a made a choice of the potentials, $\phi, \beta$, and $\gamma$, at any particular time, their subsequent evolution is uniquely determined by the equations of motion (10).

We can relate the gauge invariance to conservation laws. From

$$
\dot{\rho}+\nabla \cdot \rho \mathbf{v}=0, \quad \dot{\beta}+(\mathbf{v} \cdot \nabla) \beta=0, \quad \dot{\gamma}+(\mathbf{v} \cdot \nabla) \gamma=0,
$$

we deduce that

$$
F=\int \rho \mathcal{F}(\beta, \gamma) \mathrm{d}^{3} x
$$

is independent of time. Here $\mathcal{F}$ is an arbitrary function of the variables $\beta$ and $\gamma$ with position-independent coefficients.

Now any action that contains only first-order time derivatives defines a Poisson bracket and canonical structure. For two functionals $F_{1,2}$ of the fields $\rho, \phi, \beta, \gamma$, at time $t$ we define the Poisson bracket $\left\{F_{1}, F_{2}\right\}$ as

$$
\left\{F_{1}, F_{2}\right\}=\left.\frac{\mathrm{d} F_{2}}{\mathrm{~d} t}\right|_{F_{1}},
$$

where the subscript, $F_{1}$, on the derivative indicates that time evolution of the variables $\rho, \phi, \beta, \gamma$ is derived by varying the action:

$$
S\left[F_{1}\right]=\int \rho(\dot{\phi}+\beta \dot{\gamma}) \mathrm{d} t \mathrm{~d}^{3} x-\int F_{1}(\rho, \phi, \beta, \gamma) \mathrm{d} t .
$$

Such a Poisson bracket automatically satisfies all the usual properties, including skew symmetry and the Jacobi identity:

$$
\left\{F_{1},\left\{F_{2}, F_{3}\right\}\right\}+\left\{F_{2},\left\{F_{3}, F_{1}\right\}\right\}+\left\{F_{3},\left\{F_{1}, F_{2}\right\}\right\}=0 .
$$

In the present case the bracket becomes

$$
\left\{F_{1}, F_{2}\right\}=\int \mathrm{d}^{3} x\left(\frac{1}{\rho} \frac{\delta F_{1}}{\delta \beta(\mathbf{x})} \frac{\delta F_{2}}{\delta \gamma(\mathbf{x})}-\frac{\delta F_{1}}{\delta \phi(\mathbf{x})} \frac{\delta F_{2}}{\delta \rho(\mathbf{x})}-\frac{\beta}{\rho} \frac{\delta F_{1}}{\delta \phi(\mathbf{x})} \frac{\delta F_{2}}{\delta \beta(\mathbf{x})}-\left(F_{1} \leftrightarrow F_{2}\right)\right)
$$

and $(\rho, \phi)$, and $(\rho \beta, \gamma)$ constitute two canonically conjugate pairs, i.e.

$$
\left\{\rho(\mathbf{x}), \phi\left(\mathbf{x}^{\prime}\right)\right\}=\delta^{3}\left(\mathbf{x}-\mathbf{x}^{\prime}\right), \quad\left\{\rho \beta(\mathbf{x}), \gamma\left(\mathbf{x}^{\prime}\right)\right\}=\delta^{3}\left(\mathbf{x}-\mathbf{x}^{\prime}\right) .
$$

We now consider the conserved charge $F$ as the generator of an infinitesimal symmetry by setting

$$
\delta \phi=\{F, \phi\}=\mathcal{F}-\beta \frac{\partial \mathcal{F}}{\partial \beta} .
$$

Similarly

$$
\delta \beta=-\frac{\partial \mathcal{F}}{\partial \gamma}, \quad \delta \gamma=\frac{\partial \mathcal{F}}{\partial \beta} .
$$

The field $\rho$ is unaltered. This is because $F$ does not contain $\phi$. 
These variations generate an infinite-dimensional global (rigid, non-gauged) symmetry group. It is a global symmetry because the parameters in $\mathcal{F}$ are required to be independent of $\mathbf{x}$ and $t$. The transformations are the extension to Clebsch potentials of the global $U(1)$ phase symmetry $\phi \rightarrow \phi+$ constant which appears in potential flow, $\mathbf{v}=\nabla \phi$, where it is generated by the conserved charge $Q=\int \rho \mathrm{d}^{3} x$.

The symmetry transformations leave the Hamiltonian

$$
H=\int\left\{\frac{1}{2} \rho(\nabla \phi+\beta \nabla \gamma)^{2}+u(\rho)\right\} \mathrm{d}^{3} x
$$

invariant because $\{F, H\}=-\{H, F\}=\mathrm{d} F / \mathrm{d} t=0$. In addition to Poisson-commuting with the Hamiltonian, the conserved charge $F$ generates variations that preserve $\mathbf{v}$ itself:

$$
\delta \mathbf{v}=\nabla \delta \phi+\delta \beta \nabla \gamma+\beta \nabla \delta \gamma,=\nabla\left(\mathcal{F}-\beta \frac{\partial \mathcal{F}}{\partial \gamma}\right)-\frac{\partial \mathcal{F}}{\partial \gamma} \nabla \gamma+\beta \nabla\left(\frac{\partial \mathcal{F}}{\partial \beta}\right),=0 .
$$

They also preserve the kinetic term:

$$
\begin{aligned}
\delta[\rho(\dot{\phi}+\beta \dot{\gamma})]= & \rho\left\{\frac{\partial \mathcal{F}}{\partial \beta} \dot{\beta}+\frac{\partial \mathcal{F}}{\partial \gamma} \dot{\gamma}+\left(\frac{\partial \mathcal{F}}{\partial t}\right)_{\beta, \gamma}-\dot{\beta} \frac{\partial \mathcal{F}}{\partial \beta}-\beta\left(\frac{\partial^{2} \mathcal{F}}{\partial \beta^{2}} \dot{\beta}+\frac{\partial^{2} \mathcal{F}}{\partial \beta \partial \gamma} \dot{\gamma}+\frac{\partial^{2} \mathcal{F}}{\partial t \partial \beta}\right)\right. \\
& \left.-\frac{\partial \mathcal{F}}{\partial \gamma} \dot{\gamma}+\beta\left(\frac{\partial^{2} \mathcal{F}}{\partial \beta^{2}} \dot{\beta}+\frac{\partial^{2} \mathcal{F}}{\partial \beta \partial \gamma} \dot{\gamma}+\frac{\partial^{2} \mathcal{F}}{\partial \beta \partial t}\right)\right\}=\rho\left(\frac{\partial \mathcal{F}}{\partial t}\right)_{\beta \gamma}
\end{aligned}
$$

which vanishes provided $F$ does not explicitly depend on time.

It is easy to show that the symmetry group is that of orientation and area preserving diffeomorphisms of the 2-plane. It is equivalently the group of nonlinear canonical transformations on a two-dimensional phase space with Darboux co-ordinates $\beta, \gamma$. Because of this we can obtain the finite form of the transformations-as well as confirming that that they exhaust all transformations that preserve $\mathbf{v}$-by exploiting the familiar generating function methods from classical mechanics [21]. Suppose that

$$
\mathrm{d} \tilde{\phi}+\tilde{\beta} \mathrm{d} \tilde{\gamma}=\mathrm{d} \phi+\beta \mathrm{d} \gamma
$$

Then

$$
\mathrm{d}(\tilde{\phi}-\phi)=\beta \mathrm{d} \gamma-\tilde{\beta} \mathrm{d} \tilde{\gamma}
$$

and there must exist a $W(\gamma, \tilde{\gamma})$, the generating function, such that

$$
\tilde{\phi}-\phi=W, \quad \frac{\partial W}{\partial \gamma}=\beta, \quad \frac{\partial W}{\partial \tilde{\gamma}}=\tilde{\beta} .
$$

Conversely, given a generating function, we can obtain a finite canonical transformation. To make contact with the infinitesimal transformations we considered earlier, we let

$$
\tilde{\beta}=\beta+\dot{\beta} \Delta t, \quad \tilde{\gamma}=\gamma+\dot{\gamma} \Delta t,
$$

where ' $t$ ' is a notional time parameterizing the change. Thus

$$
\mathrm{d} W=\beta \mathrm{d} \gamma-(\beta+\dot{\beta} \Delta t) \mathrm{d}(\gamma+\dot{\gamma} \Delta t),=-\Delta t(\dot{\beta} \mathrm{d} \gamma+\beta \mathrm{d} \dot{\gamma}) .
$$

Similarly let $W=U \Delta t$, so that

$$
\mathrm{d} U=-\dot{\beta} \mathrm{d} \gamma-\beta \mathrm{d} \dot{\gamma}
$$


or, making a Legendre transformation $F=U+\beta \dot{\gamma}$ :

$$
\mathrm{d}(U+\beta \dot{\gamma})=-\dot{\beta} \mathrm{d} \gamma+\dot{\gamma} \mathrm{d} \beta=\mathrm{d} F(\beta, \gamma) .
$$

In other words

$$
\dot{\beta}=-\frac{\partial F}{\partial \gamma}, \quad \dot{\gamma}=\frac{\partial F}{\partial \beta},
$$

leading to

$$
\tilde{\phi}=\phi+U \Delta t=\phi+\left(F-\beta \frac{\partial F}{\partial \beta}\right) \Delta t, \quad \tilde{\beta}=\beta-\frac{\partial F}{\partial \gamma} \Delta t, \quad \tilde{\gamma}=\gamma+\frac{\partial F}{\partial \beta} \Delta t,
$$

as before.

\section{References}

[1] A.D. Pierce, Acoustics, Acoustical Society of America, New York, 1981.

[2] W. Unruh, Phys. Rev. Lett. 46 (1981) 1351.

[3] W. Unruh, Phys. Rev. D 51 (1995) 2827. gr-qc/9409008.

[4] M. Visser, Class. Quant. Gravit. 15 (1998) 1767-1791. gr-qc/9712010.

[5] M. Visser, Lecture at the 1998 Peñiscola Summer School on Particle Physics and Cosmology. gr-qc/9901047.

[6] http://www.mcs.vuw.ac.nz/ visser/Analog. Mirror sites at http://www.physics.wustl.edu/ visser/Analog and http://www.cbpf.br/ bscg/analog/.

[7] M. Stone, Phys. Rev. E 62 (2000) 1341. cond-mat/9909315.

[8] M. Stone, Phonons and forces: momentum versus pseudomomentum in moving fluids, in: M. Novello, G. Volovik, M. Visser (Eds.), Artificial Black Holes, World Scientific, Singapore, 2002. cond-mat/0012316.

[9] A.D. Pierce, J. Acoust. Soc. Am. 87 (1990) 2292.

[10] D.I. Blokhintsev, Acoustics of a Non-homogeneous Moving Medium, Gostekhizdat, 1945 (English translation: N.A.C.A. Technical Memorandum no. 1399 (1956)).

[11] D. Blokhintzev, J. Acoust. Soc. Am. 18 (1946) 322-328.

[12] H. Bateman, Proc. Roy. Soc. London A 125 (1929) 598-618.

[13] C.C. Lin, Liquid helium, in: Proceedings of the International School of Physics, "Enrico Fermi", Course XXI, Academic Press, New York, 1965.

[14] R.L. Seligar, G.B. Whitham, Proc. Roy. Soc. London A 305 (1968) 1-25.

[15] R.F.A. Clebsch, J. Reine Angew. Math. ("Crelle") 56 (1859) 1.

[16] Sir Horace Lamb, Hydrodynamics, Dover, New York, 1945, p. 248.

[17] W. Möhring, Energy flux in duct flow, J. Sound Vib. 18 (1971) 101.

[18] H. Cendra, J.E. Marsden, Lin constraints, Clebsch potentials and variational principles, Physica D 27 (1987) 63-89; J.E. Marsden, A. Weinstein, Coadjoint orbits, vortices, and Clebsch variables for incompressible fluids, Physica D 7 (1983) $305-323$.

[19] S. Bahcall, L. Susskind, Fluid dynamics, Chern-Simons theory and the quantum Hall effect, Int. J. Mod. Phys. B 5 (1991) 2735 ; L. Susskind, The quantum Hall fluid and non-commutative Chern-Simons theory. arXiv:hep-th/0101029.

[20] M.S. Howe, Acoustics of Fluid-Structure Interactions, Cambridge University Press, Cambridge, 1998.

[21] H. Goldstein, Classical Mechanics, Addison-Wesley, Reading, MA, 1980.

[22] R. Arnowitt, S. Deser, C.W. Misner, in: L. Witten (Ed.), Gravitation: An Introduction to Current Research, Wiley, NY, 1962, pp. 227-265; C. Misner, K. Thorne, J. Wheeler, Gravitation, Freeman, San Francisco, 1973.

[23] C. Misner, K. Thorne, J. Wheeler, Gravitation, Freeman, San Francisco, 1973, p. 504

[24] E.B. Sonin, Vortex oscillations and hydrodynamics of rotating superfluids, Rev. Mod. Phys. 59 (1987) 87 (see especially, pp. 90-91).

[25] Sir James Lighthill, Waves in Fluids, Cambridge University Press, Cambridge, 1978.

[26] G.I. Taylor, Proc. Roy. Soc. A 102 (1922) 180-189; G.I. Taylor, Proc. Roy. Soc. A 104 (1923) 213-218.

[27] J. Proudman, Proc. Roy. Soc. A 92 (1916) 408-424.

[28] J. Pedlosky, Geophysical Fluid Dynamics, Springer-Verlag, 1986. 